\title{
Ice Accretion Measurements on an Airfoil and Wedge in Mixed-phase Conditions
}

\author{
Peter Struk \\ NASA GRC, Cleveland, Ohio, USA \\ Tadas Bartkus, Jen-ching Tsao \\ NASA GRC, Ohio Aerospace Institute, Cleveland, Ohio, USA \\ Tom Currie, Dan Fuleki \\ National Research Council of Canada, Ottawa, Ontario, Canada
}

Presented at the SAE 2015 International Conference

on Icing of Aircraft, Engines, and Structures

Prague, Czech Republic, June 22-25, 2015 


\section{Outline}

- Background

- Introduction

- Experimental description

- Test conditions

- Imaging \& analysis method

- Results

- NACA 0012

- Wedge

- Summary

- Acknowledgements 


\section{Background / Introduction}

- Ingestion of atmospheric ice crystals by aircraft engines can cause ice to accrete on internal components leading to rollback, flameout, mechanical damage, etc.

- Experiments underway to understand fundamentals of mixed-phase and ice crystal icing in jet engines

- NASA \& NRC collaborations have had 3 test entries:

- Nov 2010

- Mar 2011

- Mar \& Apr 2012

- Traditional methods of recording ice shapes (e.g. tracings and castings) were not easily adaptable to this experiment

- Rely primarily on video imagery

- First two test methods only captured 1D ice growth along leading edge

- 2012 test entry produced first analyzable 2D shapes

- This paper presents the ice accretion shape and surface temperature measurements from the 2012 test entry

- The measurements are intended to help develop models of the ice-crystal icing phenomenon associated with engine ice-crystal icing

- However, the primary test objectives of the 2012 entry were to characterize facility and prove out imaging concepts so only limited test runs were dedicated for accretion 


\section{Experiment Overview}

NRC's Research Altitude Test Facility (RATFac)

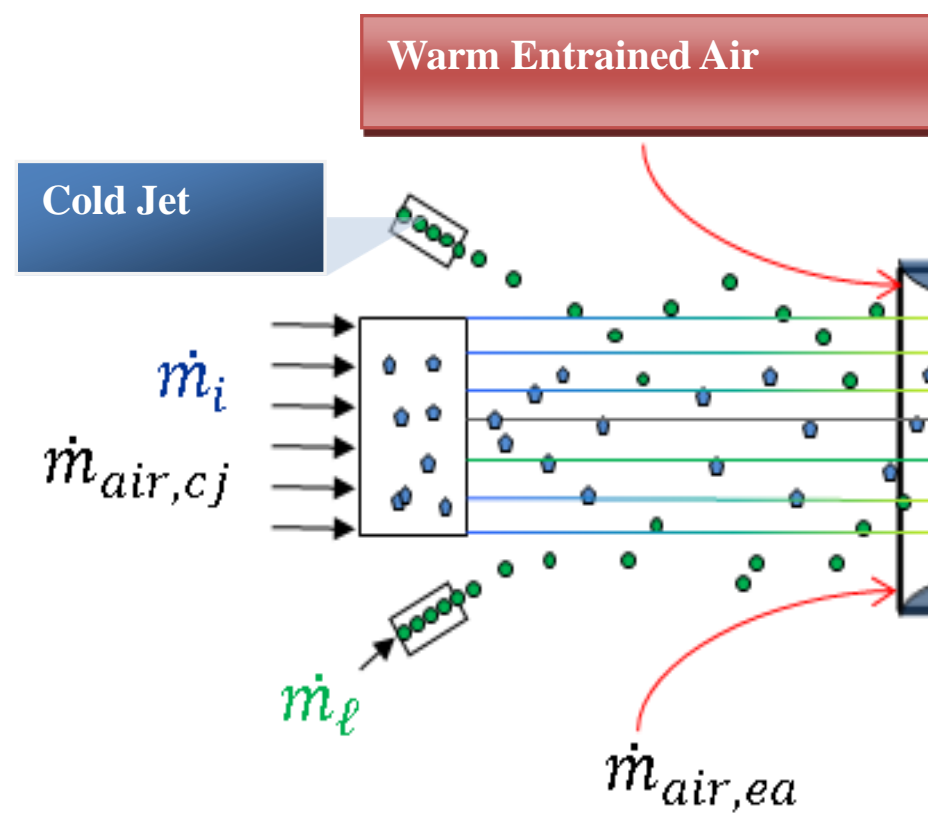

Test Procedure:

1. Set dry (cloud off) conditions

2. Turn on cloud for $\sim 3-5$ min for accretion test

3. Inserted SEA multiwire for LWC / TWC measurements
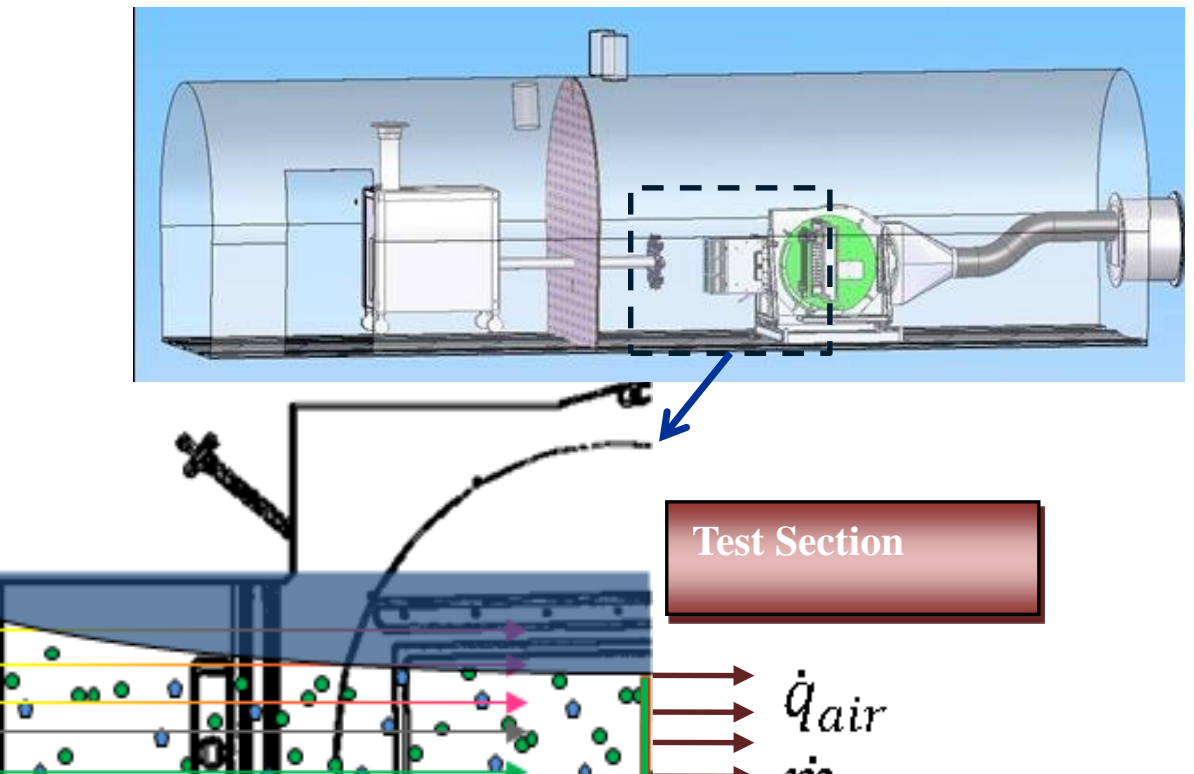

$\dot{m}_{\text {air }}$

$U, T_{0} P_{0}$

$\mathrm{SH}, \mathrm{RH} \%$

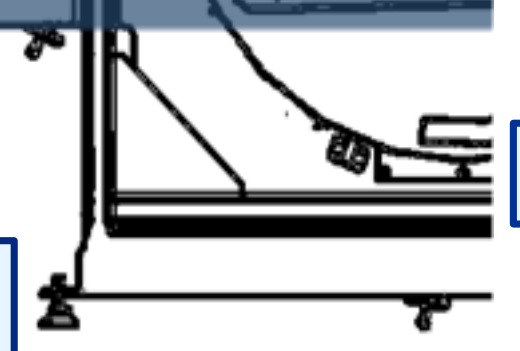

Cascade Rig 


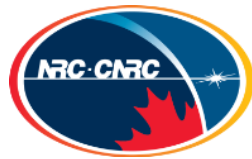

\section{Test Articles}

\section{NACA 0012 Airfoil}

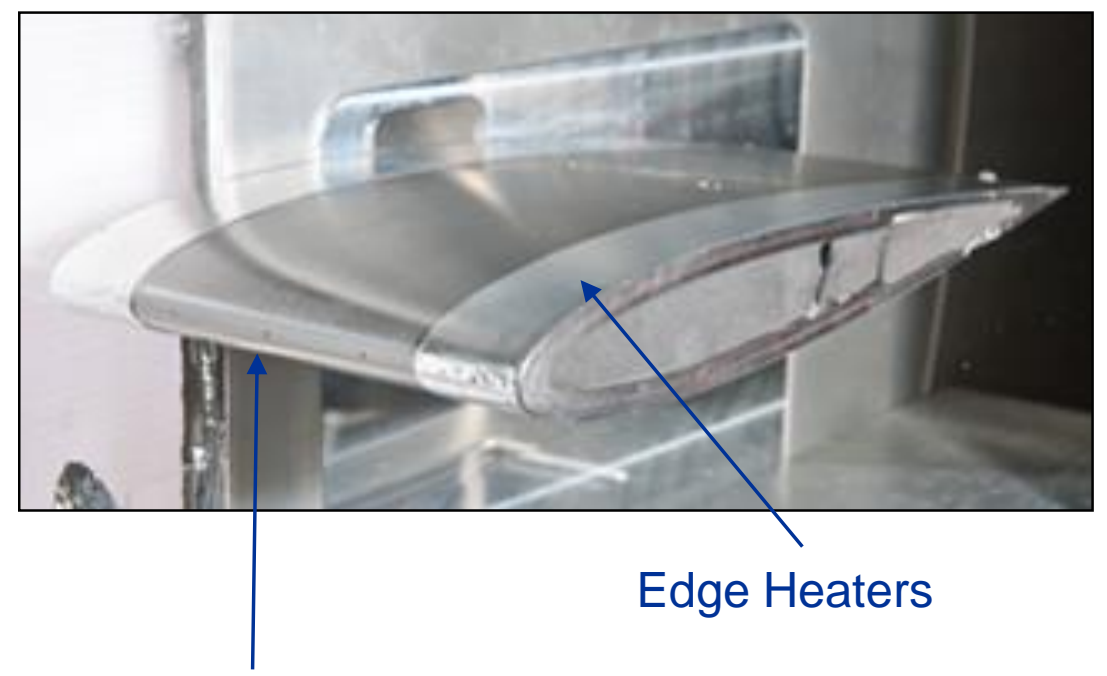

Thermocouples (x 15)

\section{Wedge Airfoil}

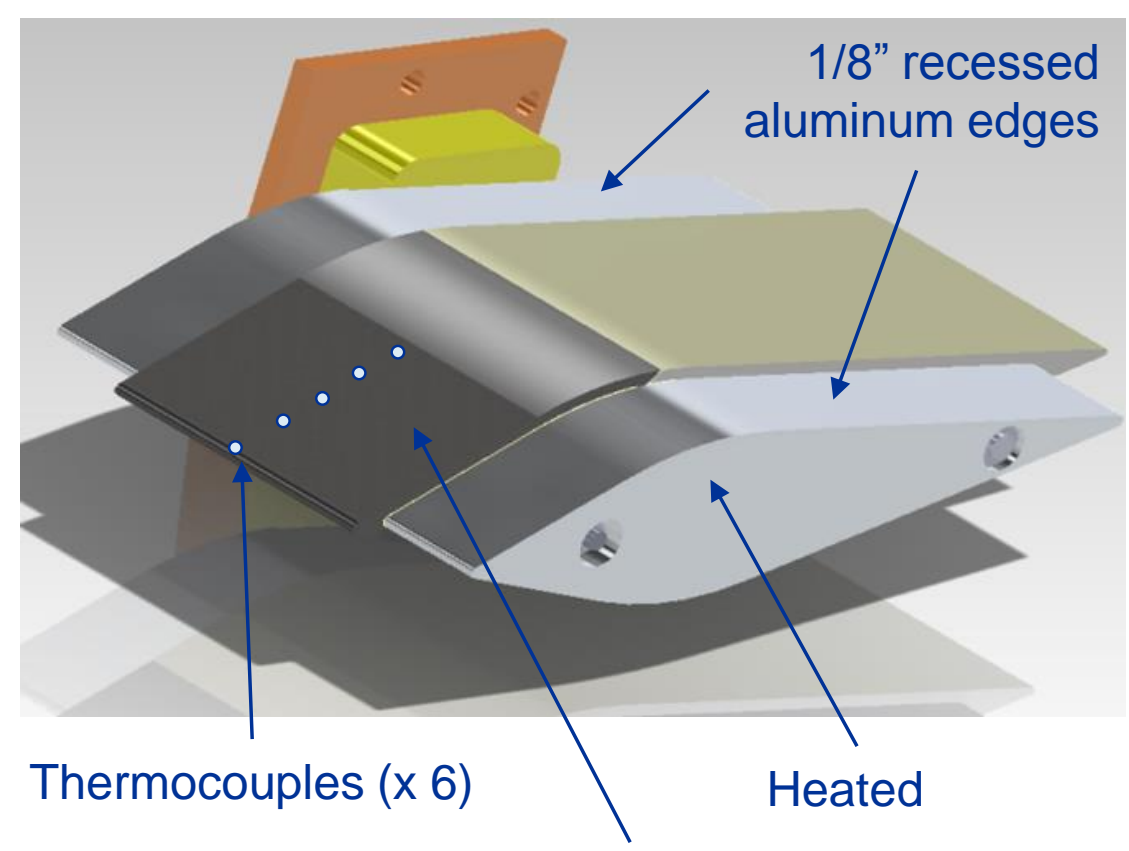

Heated / Cooled Surface

(described later) 


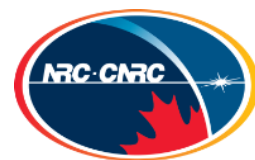

\section{Camera Setup - 2012}
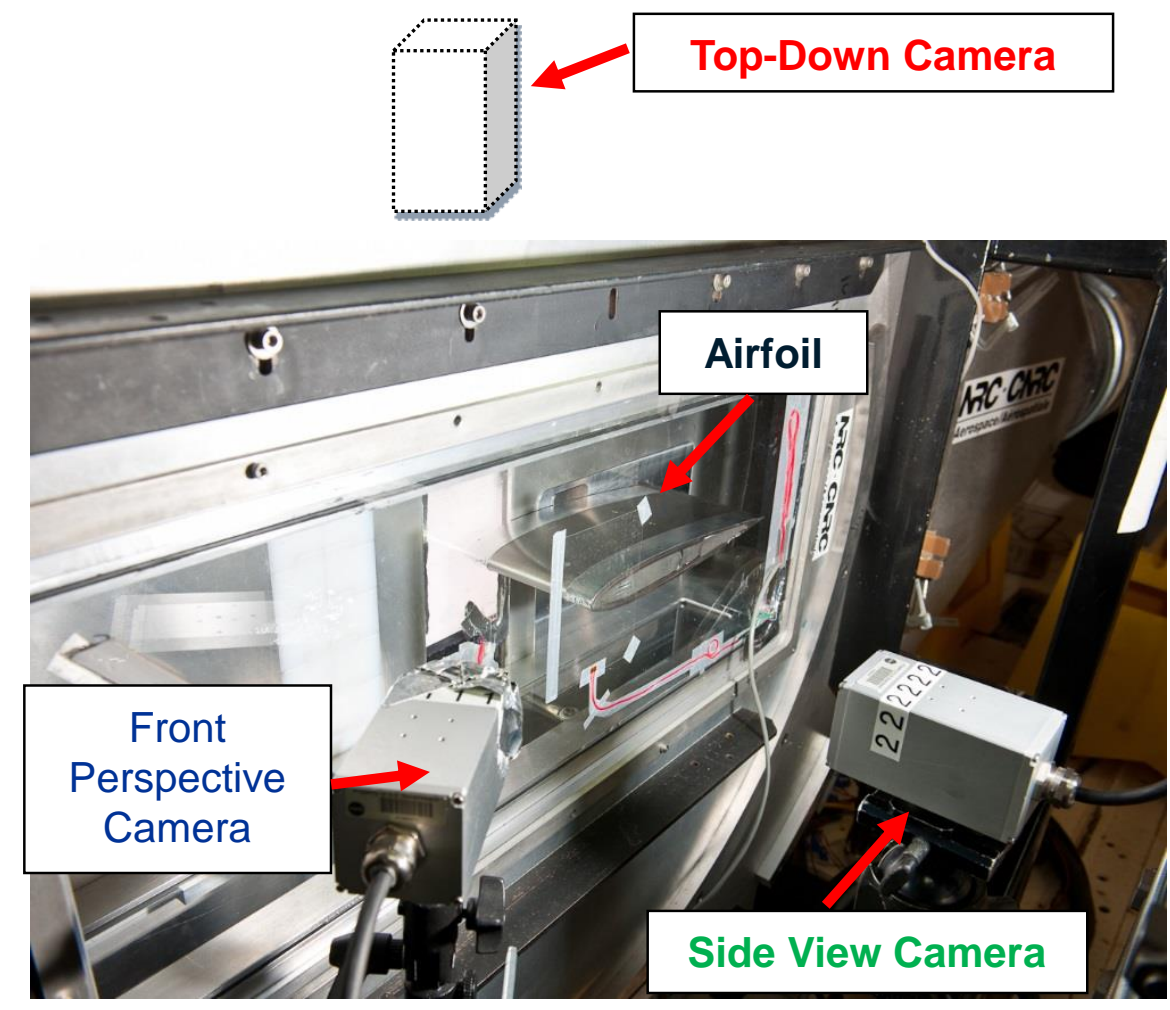

Side view could not be analyzed from NACA 0012 cases due to obscurations

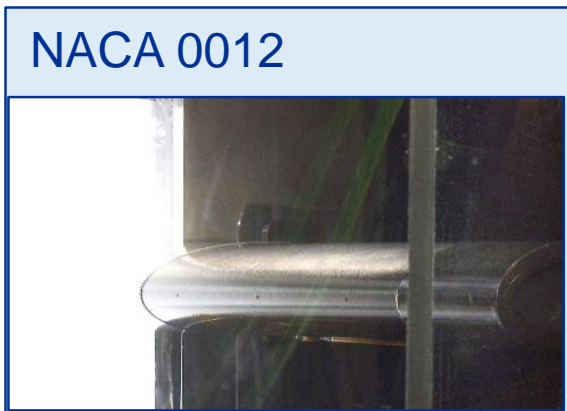

Front Perspective View

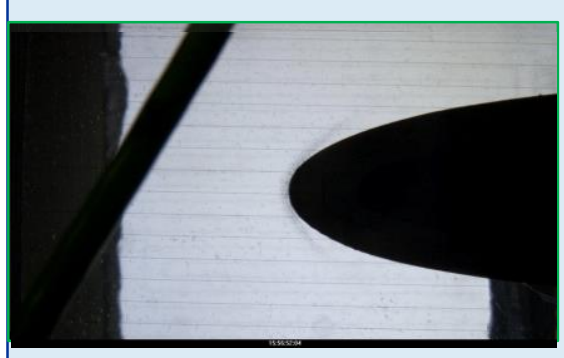

Side View

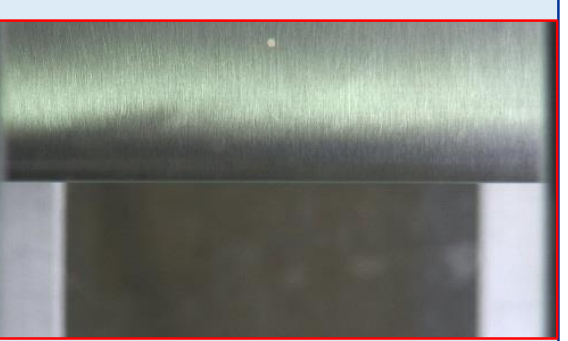

Top Down View

Test 824

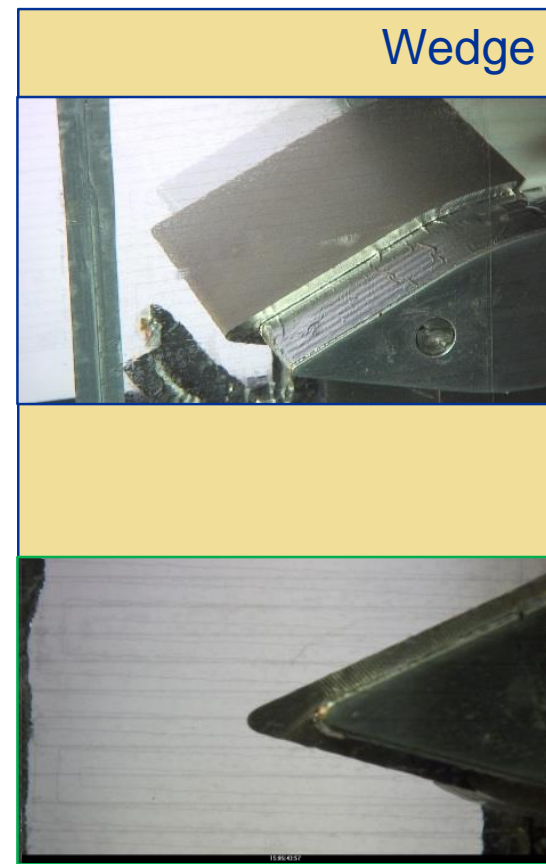

24X playback

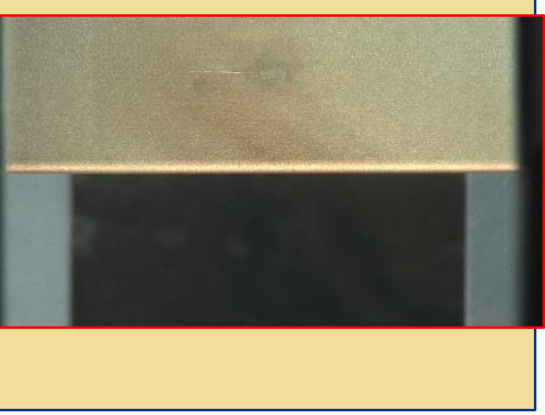

Test 1003 


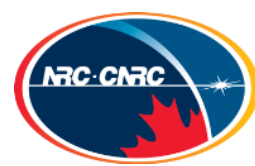

\section{Optical View Port Tests}

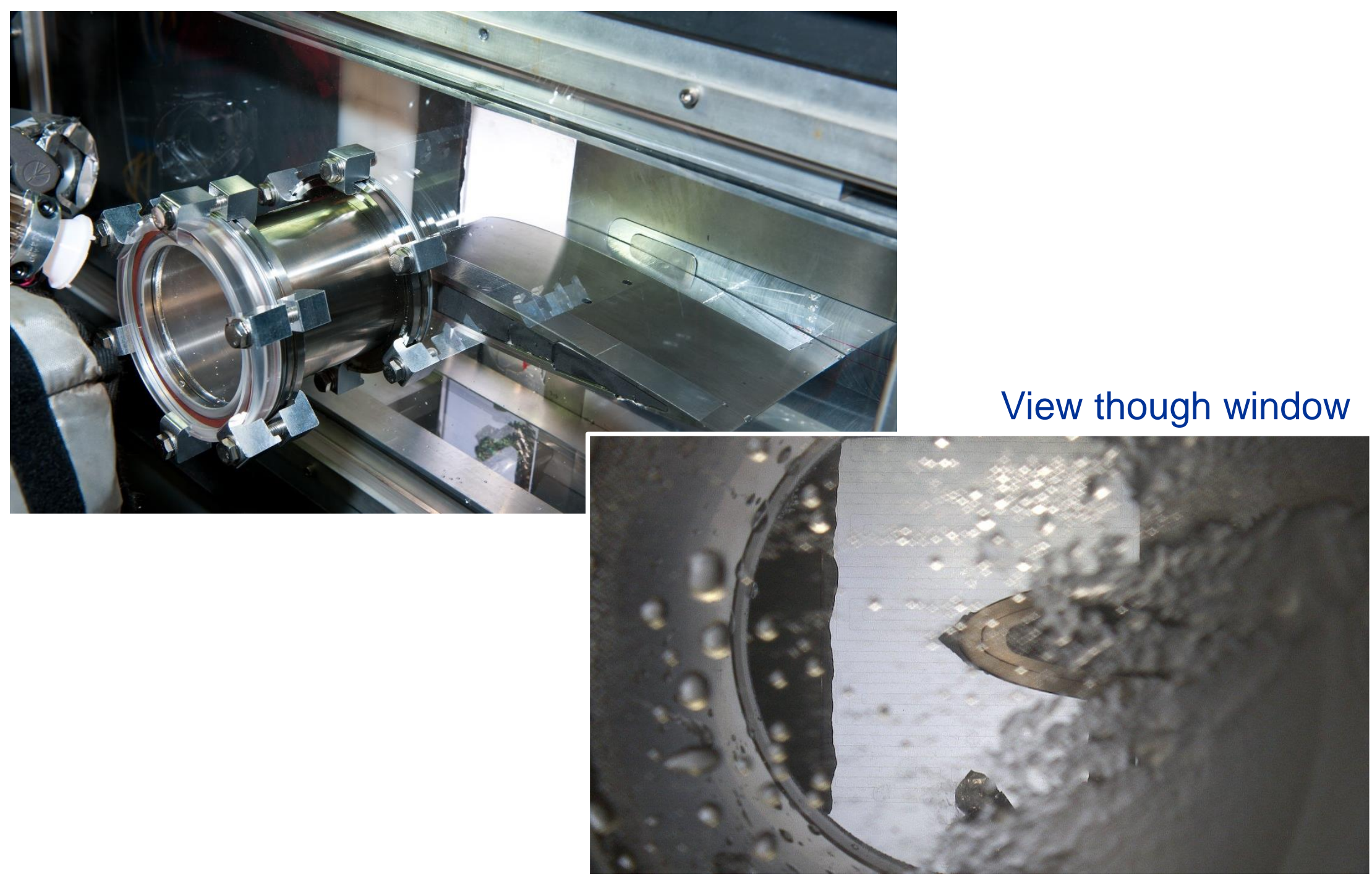




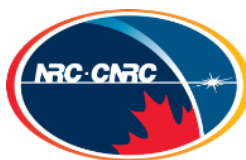

\section{Analysis}

Clean
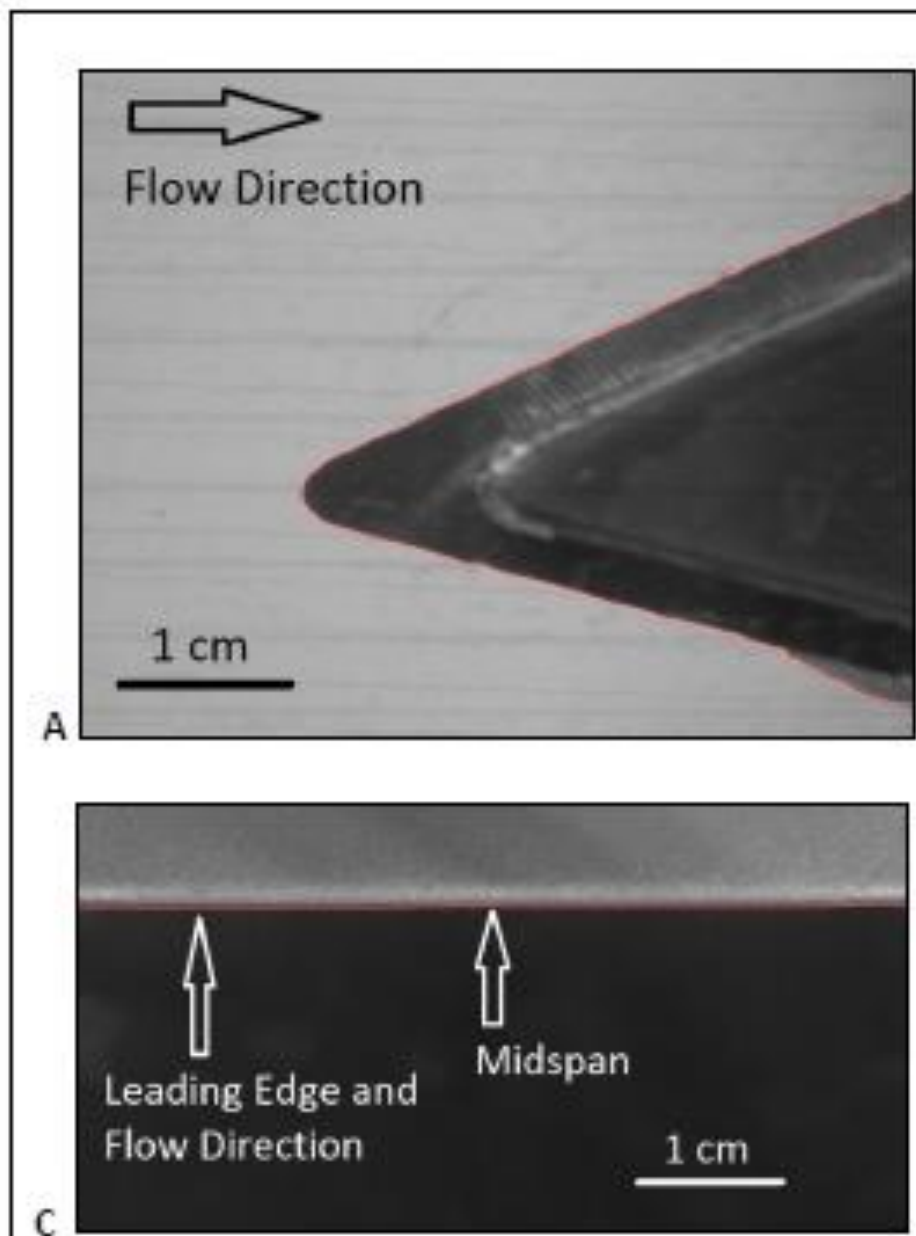

Iced
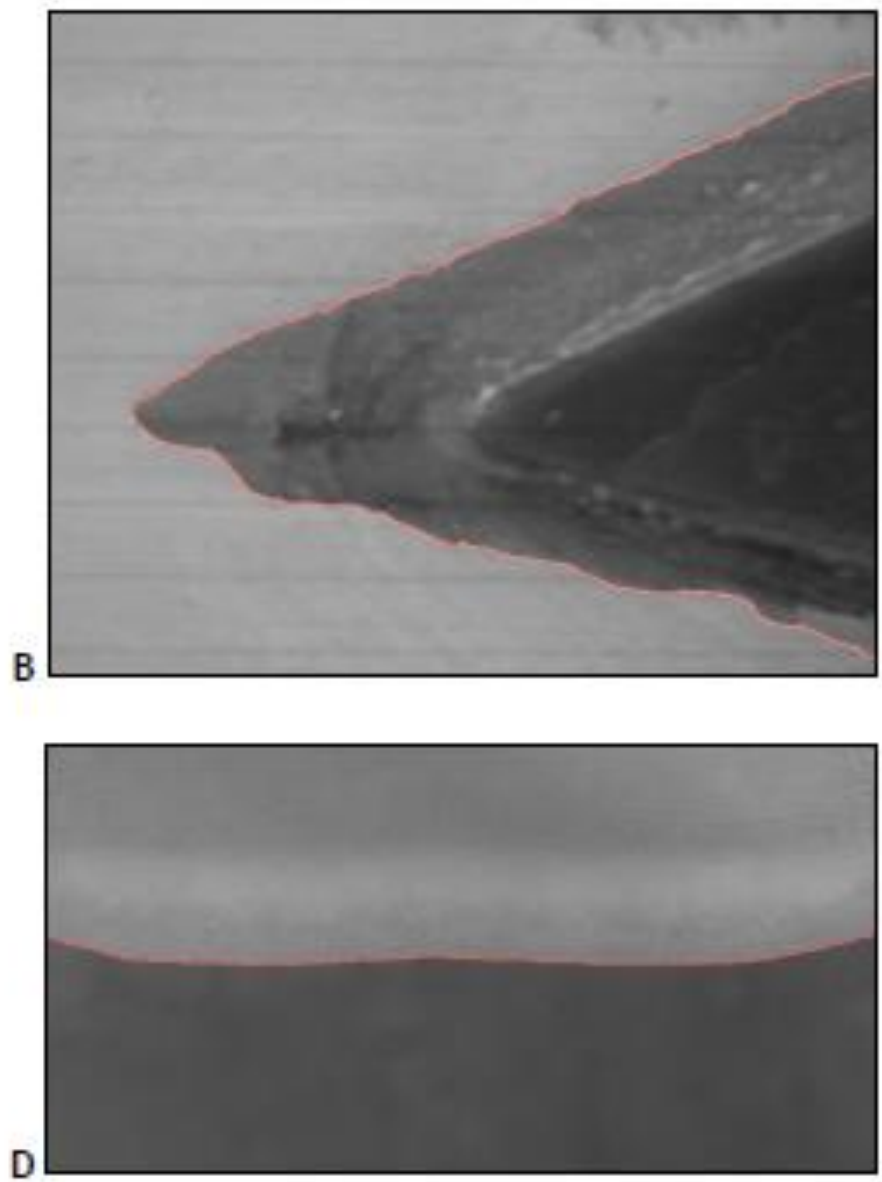


\section{Test Conditions - NACA 0012}

- $\boldsymbol{P}_{0}=6.5$ or 4.0 psia $(\sim 45 \& 28 \mathrm{kPa})$

- $\boldsymbol{U}=85$ or $135 \mathrm{~m} / \mathrm{s}$

- $T_{0}=\sim 6$ to $19^{\circ} \mathrm{C}$ (cloud off)

- Decreased with cloud on

- Wet bulb: $-5^{\circ} \mathrm{C}<T_{w b}<4$

- Injected steam set with cloud off

- Measured humidity cloud off \& on

- $\quad I W C_{\mathrm{i}}=7 \mathrm{~g} / \mathrm{m}^{3}$ (no supplemental water)

\begin{tabular}{|c|c|c|c|c|c|c|}
\hline$\stackrel{\vec{y}}{\vec{E}}$ & 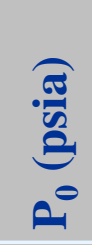 & $\begin{array}{l}\tilde{n} \\
\text { ह्छ } \\
D\end{array}$ & $\underbrace{0}_{\substack{0 \\
0}}$ & $\underbrace{0}_{0}$ & $\underbrace{0}_{0}$ & $\underbrace{0}_{\substack{0 \\
0}}$ \\
\hline 796 & 6.5 & 86.2 & 13.2 & 7.2 & -0.3 & -0.1 \\
\hline 867 & 4.0 & 134.4 & 18.0 & 9.1 & 2.1 & 1.7 \\
\hline
\end{tabular}

$T W C_{t}=C F_{i c e} I W C_{i}+C F_{n o z} L W C_{i}-\left(G W C_{o n}-G W C_{o f f}\right)$

\begin{tabular}{c|c|c|}
\hline $\begin{array}{c}\text { Water } \\
\text { source }\end{array}$ & $\begin{array}{c}\text { Velocity } \\
(\mathbf{m} / \mathbf{s})\end{array}$ & \multicolumn{1}{c|}{ CF } \\
\hline $\begin{array}{c}\text { LWC } \\
\text { (nozzles) }\end{array}$ & all & $C F_{\text {noz }}=1.0$ \\
\hline $\begin{array}{c}\text { IWC } \\
\text { (grinder) }\end{array}$ & 85 & $C F_{\text {ice }}=1.0$ \\
\hline
\end{tabular}

$M R=\frac{\max \left(L W C_{m, 083}, L W C_{m, 021}\right)}{T W C_{t}}$

\begin{tabular}{|c|c|c|c|c|c|c|c|}
\hline$\stackrel{\bar{g}}{\Leftrightarrow}$ & 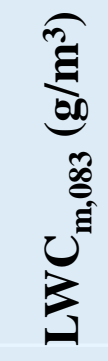 & 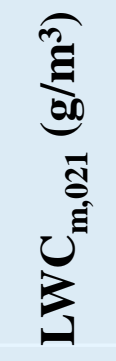 & 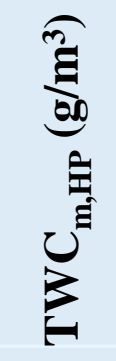 & 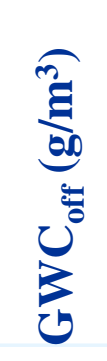 & 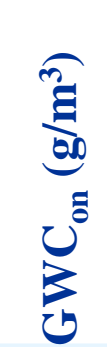 & 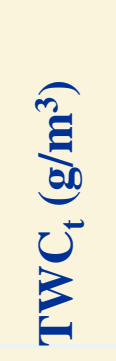 & 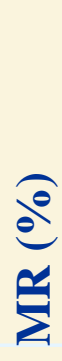 \\
\hline 796 & 0.67 & 0.51 & 3.58 & 1.84 & 3.21 & 5.54 & 12 \\
\hline 867 & 0.92 & 1.29 & 4.85 & 3.35 & 4.01 & 9.13 & 14 \\
\hline
\end{tabular}


NACA 0012 Result - Test 796

$$
U=85 \mathrm{~m} / \mathrm{s}, P_{0}=6.5 \text { psia, } T_{\text {wbo }}=-0.1^{\circ} \mathrm{C}, \mathrm{IWC}_{\mathrm{i}} \cong 7 \mathrm{~g} / \mathrm{m}^{3}
$$
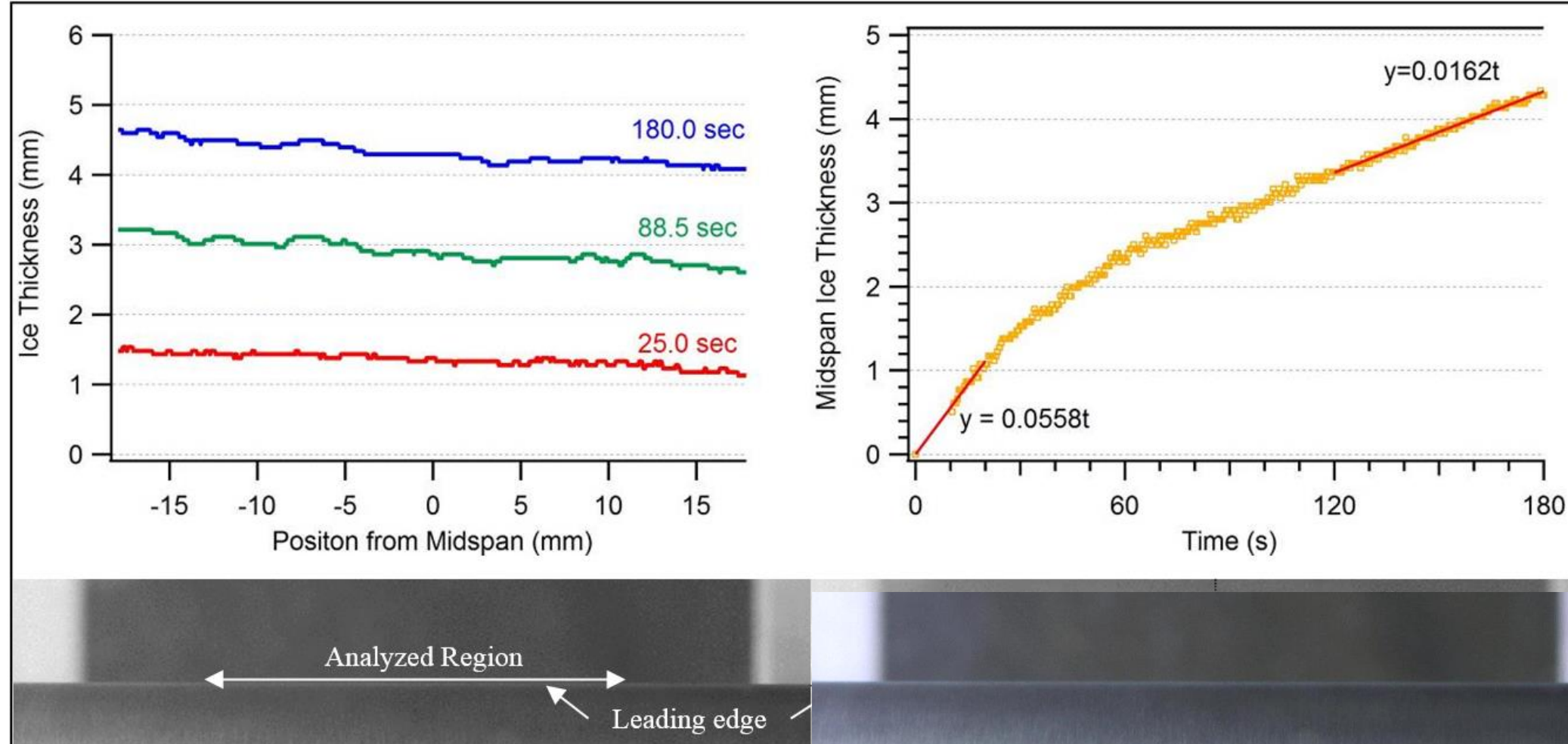


\section{Variation of growth rate with MR}

\begin{tabular}{|cl|}
\hline Parameter & Value \\
\hline$U$ & $85 \mathrm{~m} / \mathrm{s}$ \\
\hline $\mathrm{P}_{0}$ & $6.5 \mathrm{psia}$ \\
\hline $\mathrm{IWC}_{\mathrm{i}}$ & $7 \mathrm{~g} / \mathrm{m}^{3}$ \\
\hline $\mathrm{T}_{\mathrm{WB}}$ & 0 to $-1^{\circ} \mathrm{C}$ \\
\hline
\end{tabular}

Scans 824, 802, 834, 796

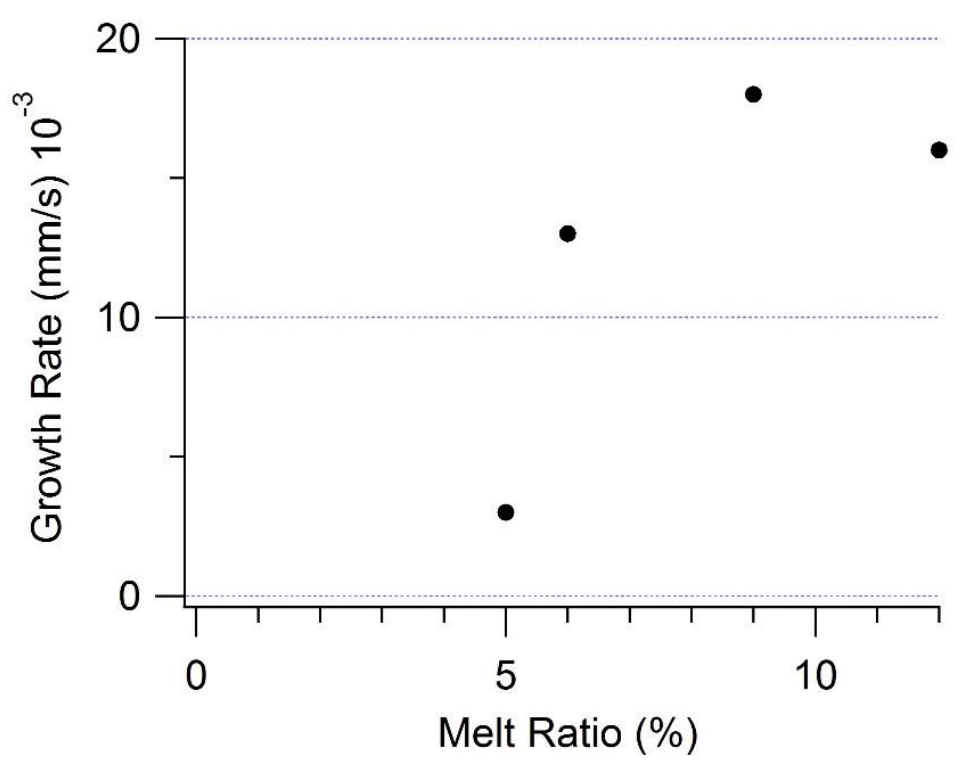

$\dot{r}$ increases then decreases with MR 
$U=134 \mathrm{~m} / \mathrm{s}, P_{0}=4.0$ psia, $T_{\text {wb0 }}=1.7^{\circ} \mathrm{C}, \mathrm{IWC}_{\mathrm{i}} \cong 7 \mathrm{~g} / \mathrm{m}^{3}, \mathrm{MR}=14 \%$
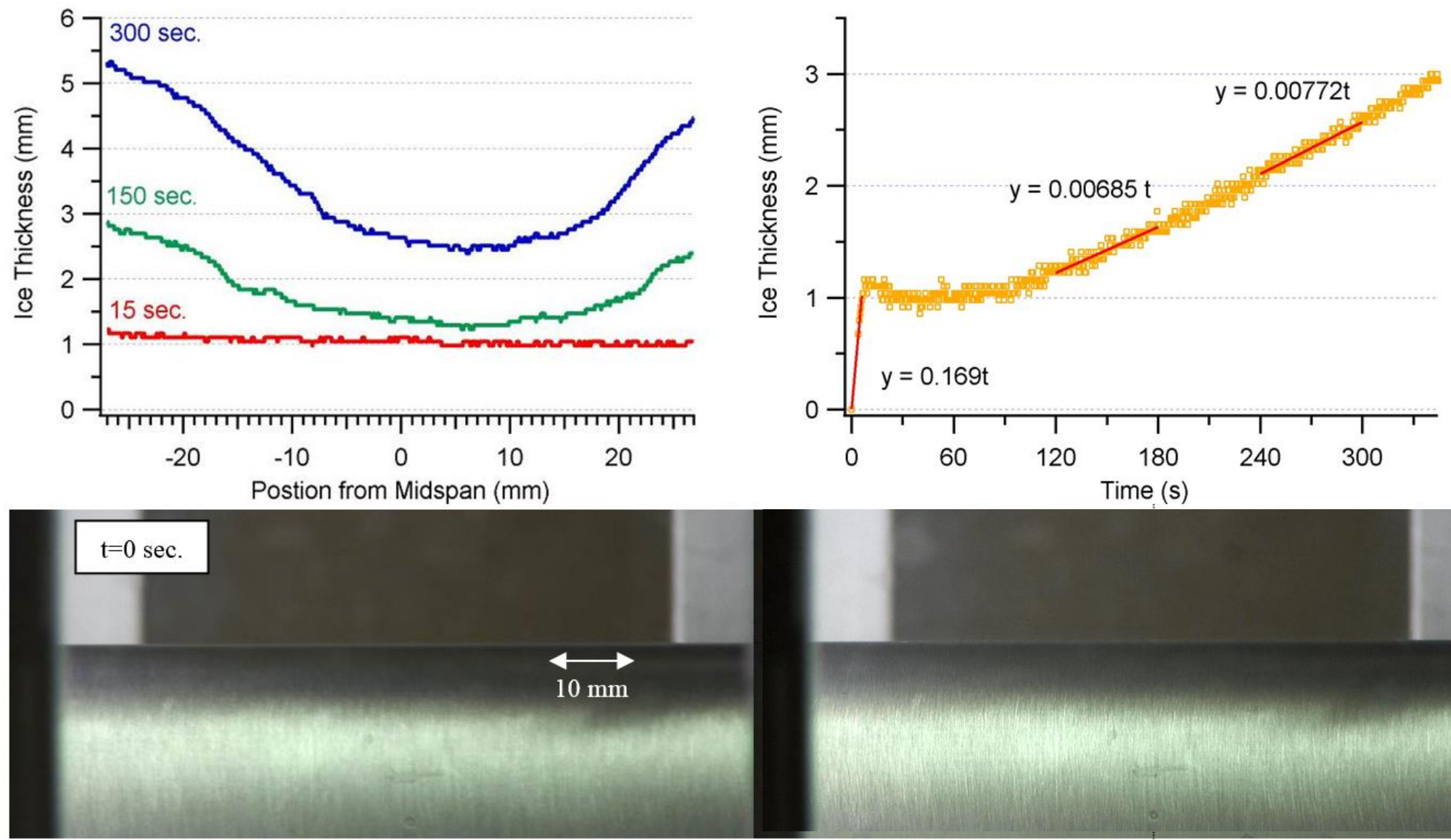

Only case with substantial ice growth at $135 \mathrm{~m} / \mathrm{s}$ from available data

24X playback 


\section{Test Conditions - Wedge}

- $\boldsymbol{P}_{\boldsymbol{0}}=6.5$ or 10.0 psia $(\sim 45 \& 69 \mathrm{kPa})$

- $\boldsymbol{U}=85 \mathrm{~m} / \mathrm{s}$

- $T_{0}=\sim 8$ to $21^{\circ} \mathrm{C}$ (cloud off)

- Decreased with cloud on

- Wet bulb: $1^{\circ} \mathrm{C}<\boldsymbol{T}_{\boldsymbol{w b}}<5$

- Adjusted incoming stream and measured humidity

- $I W C_{\mathrm{i}}=\sim 7,8.5$, or $17 \mathrm{~g} / \mathrm{m}^{3}$

- One case with supplemental water (1003) 
$U=87 \mathrm{~m} / \mathrm{s}, P_{0}=6.5 \mathrm{psia}, \mathrm{T}_{\text {wb0 }}=2.5^{\circ} \mathrm{C}, \mathrm{IWC}_{\mathrm{i}} \cong 16.7 \mathrm{~g} / \mathrm{m}^{3}, \mathrm{MR}=13 \%$
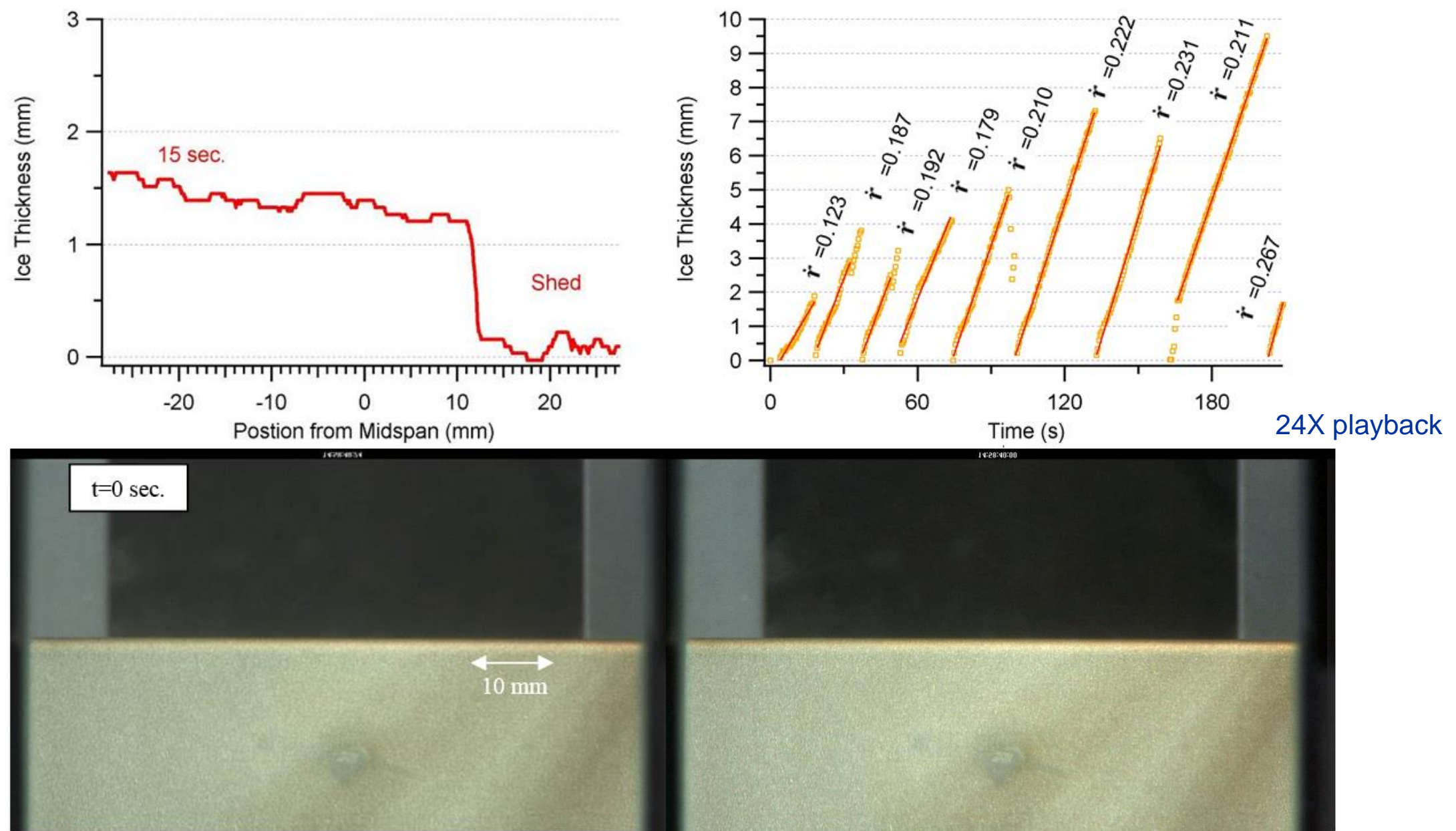

Similar test (883) with $\mathrm{IWC}_{\mathrm{i}} \cong 7 \mathrm{~g} / \mathrm{m}^{3}(\mathrm{MR}=16 \%)$ did not show ice accretion 


\section{Wedge Heating-Cooling System}

- Heating/cooling achieved by spraying heated/cooled water/antifreeze on back of 1/8" thick Ti 6Al-4V icing surface through 100 1/32" holes
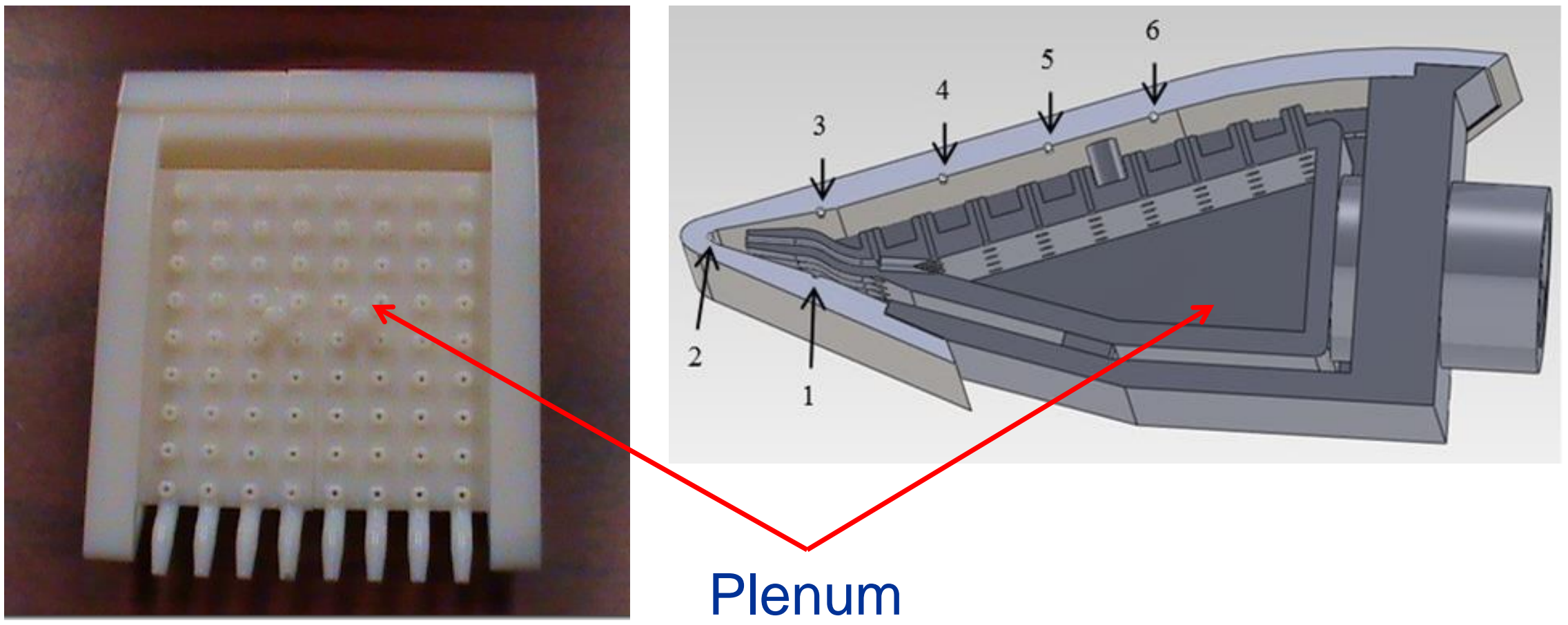


\section{Cooled Wedge Result - Test 982}

$\mathrm{U}=86 \mathrm{~m} / \mathrm{s}, P_{0}=10 \mathrm{psia}, T_{\text {wb } 0}=1.2^{\circ} \mathrm{C}, I W C_{i} \cong 16.7 \mathrm{~g} / \mathrm{m}^{3}$
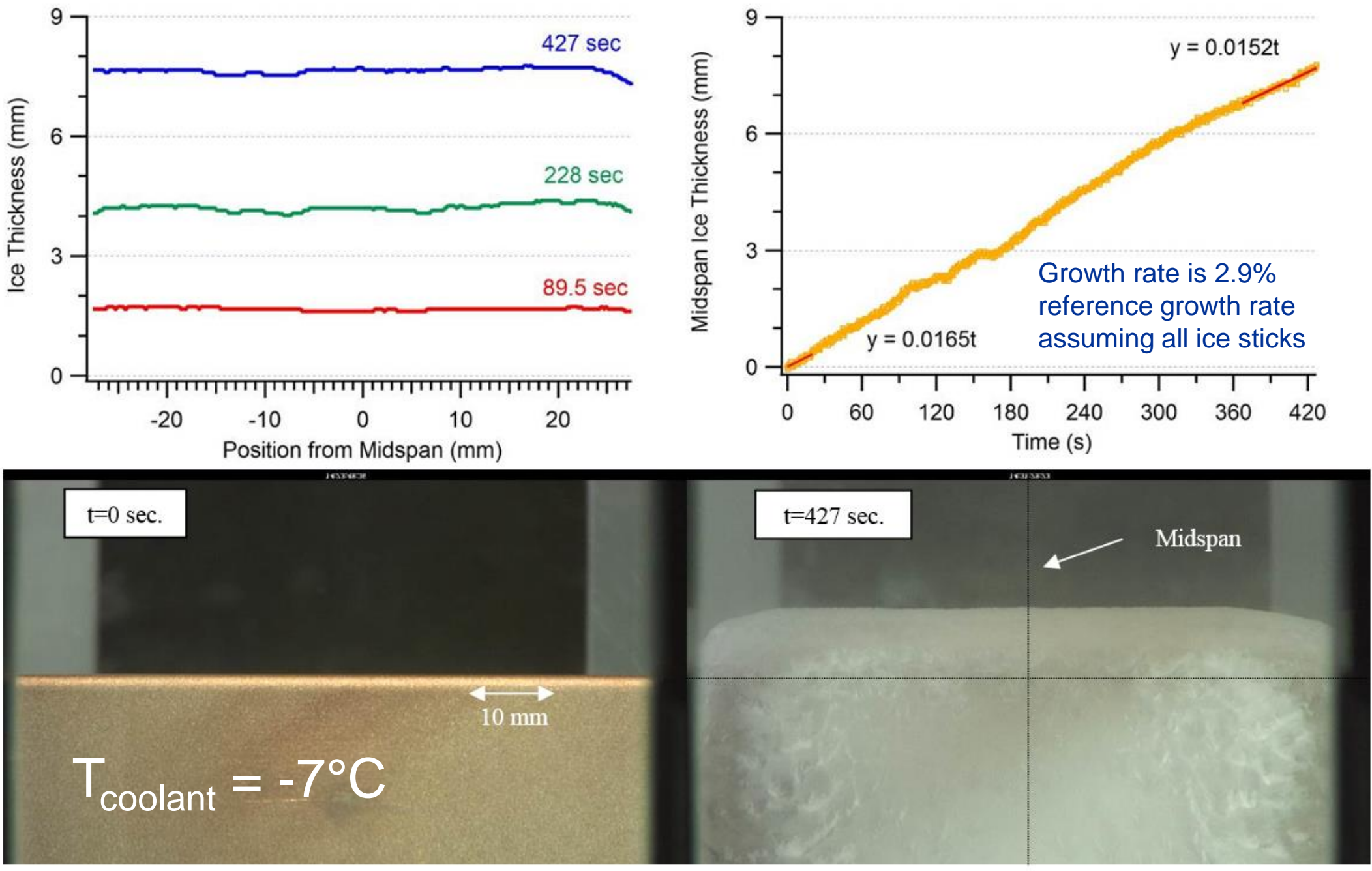
National Aeronautics and Space Administration

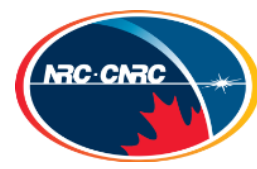

\section{Cooled Wedge Result - Test 982 (Side Profile Measurements)}

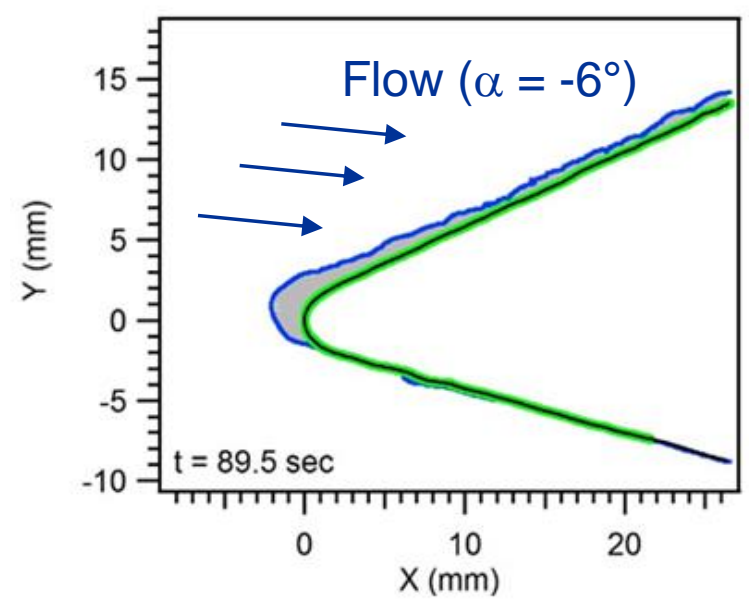

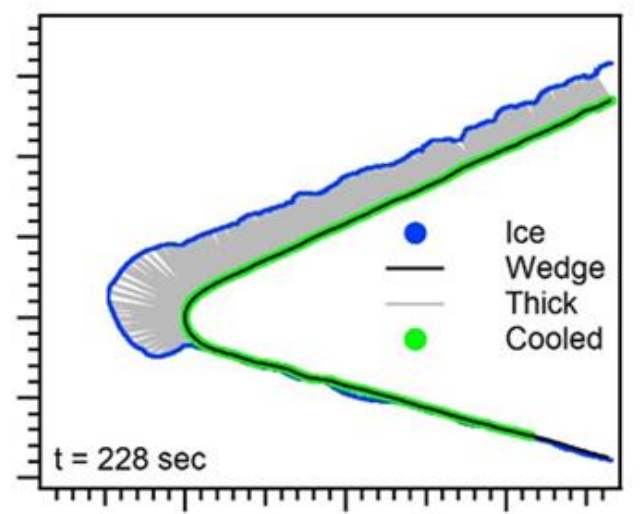

0$$
20
$$

$$
\begin{gathered}
10 \\
X(\mathrm{~mm})
\end{gathered}
$$

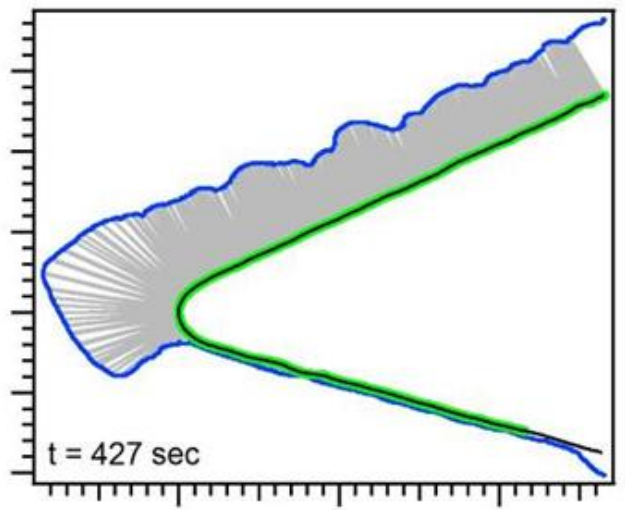

20

10
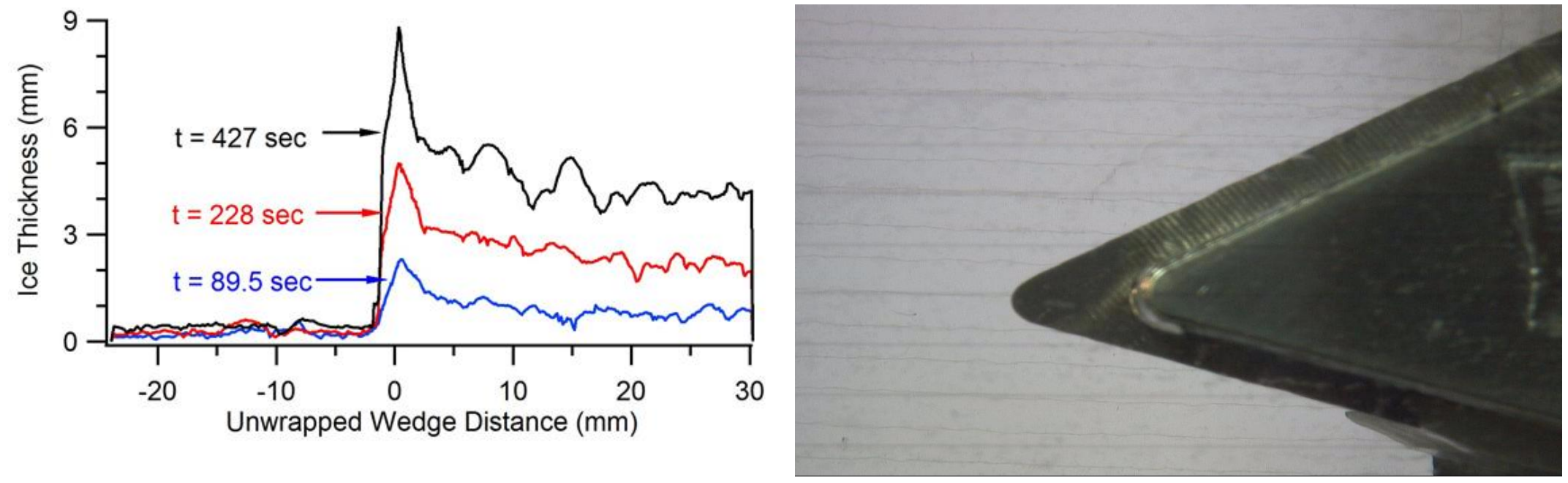

24X playback 


\section{Summary (1 of 2$)$}

- This paper presents measurements of ice accretion shape from ice-crystal icing experiments conducted at the NRC RATFac

- Data provided for development of ice-crystal accretion models

- Select surf. temperature measurements available in paper

- Used two different models: NACA 0012 and wedge

- Several wedge tests included actively cooled surface

- Tested at different U, T, and P

- Only a limited set of permutations

- NACA 0012 tests used only injected ice particles which naturally melted in the warm airflow (no supplemental LWC) 


\section{Summary (2 of 2$)$}

- The ice accretion measurements included:

- leading-edge thickness (both models)

- 2D cross-section profile (wedge \& 1 NACA 0012 case)

- NACA 0012

- In some cases, initial growth rate, $\dot{r}$, higher than at end of test

- Results suggest that $\dot{r}$ increases then decreases with MR

- $135 \mathrm{~m} / \mathrm{s}$ case showed less growth near midspan compared with root \& tip

- Wedge

- With adiabatic model, observed weakly adhered rapid accretions with shedding at $\mathrm{T}_{\mathrm{wb}}$ above freezing

- With active surface cooling, ice accretion without shedding occurred and the growth rate increased with $M R$

- Accretions grew generally parallel to the icing surface 


\section{Acknowledgements}

- This work was performed under an agreement between NASA and the National Research Council of Canada

- Financial support:

- Atmospheric Environment Safety Technologies (AEST) project under NASA's Aviation Safety Program

- National Research Council

- Federal Aviation Administration

- Transport Canada

- Special thanks are extended to Dr. Ron Colantonio, Mr. Jim MacLeod, and Mr. Thomas Bond for their support of the work

- Finally, the authors would like to thank Mr. Chris Lynch for his excellent imaging work during the experiments 


\section{Question}

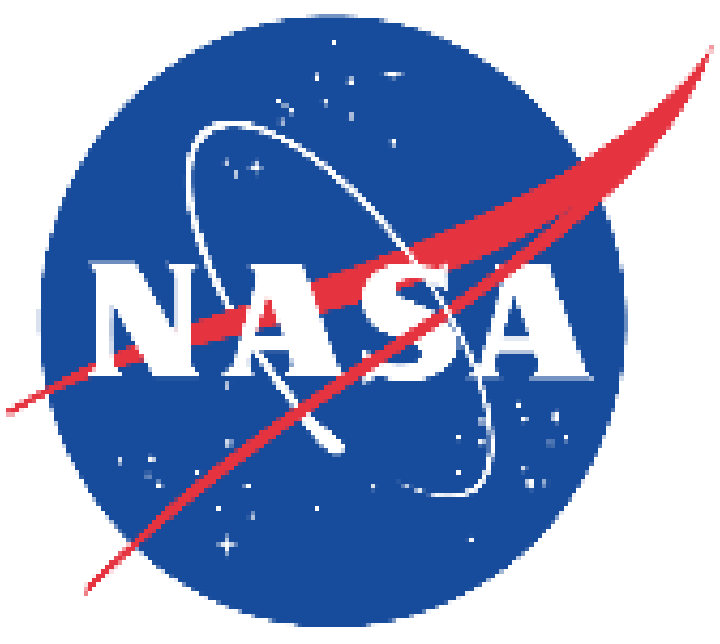


Ice Accretion Measurements on an Airfoil and Wedge in Mixed-phase Conditions BACKUP 


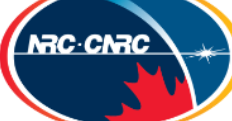

\section{Test Conditions - NACA 0012}

\begin{tabular}{|c|c|c|c|c|c|c|c|c|c|c|c|c|c|c|c|c|c|c|c|c|c|}
\hline $\begin{array}{l}\overrightarrow{v_{0}} \\
\stackrel{\theta}{*}\end{array}$ & 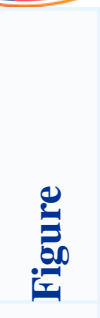 & $\underbrace{\stackrel{\sigma}{\tilde{E}}}_{0}$ & 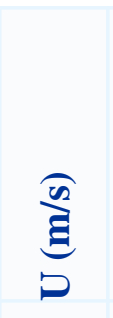 & 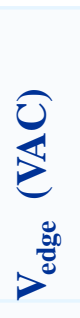 & $\begin{array}{c}\underbrace{0}_{0} \\
0 \\
0 \\
0\end{array}$ & $\underbrace{0}_{\tilde{\sigma}} \underbrace{0}$ & $\underbrace{0}_{\substack{0 \\
0}}$ & $\underbrace{0}_{\overline{0}}$ & 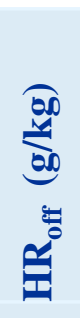 & 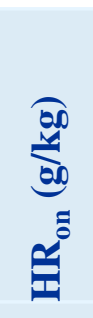 & 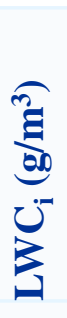 & 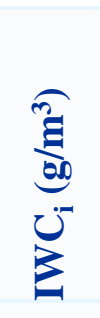 & 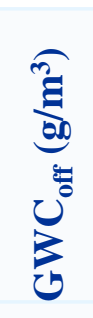 & 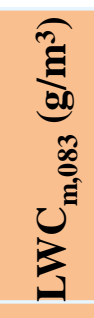 & 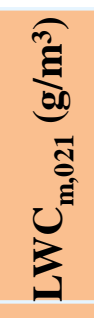 & 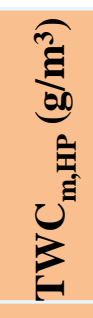 & 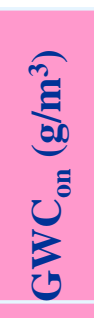 & 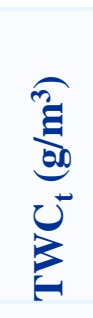 & 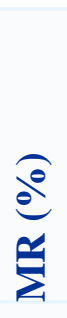 & 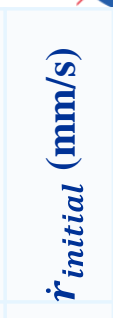 & 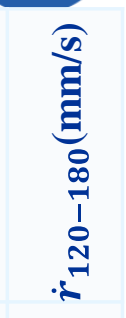 \\
\hline 796 & 6 & 6.5 & 86.2 & 0 & 13.2 & 7.2 & -0.3 & -0.1 & 3.4 & 5.9 & 0 & 6.91 & 1.84 & 0.67 & 0.51 & 3.58 & 3.21 & 5.54 & 12 & 0.056 & 0.016 \\
\hline 802 & 7 & 6.5 & 85.7 & 60 & 13.1 & 7.5 & -0.9 & -0.7 & 2.8 & 5.1 & 0 & 6.95 & 1.42 & 0.33 & 0.29 & 2.47 & 2.61 & 5.76 & 6 & 0.14 & 0.013 \\
\hline 818 & N/A & 6.5 & 84.4 & 0 & 5.8 & 0.8 & -4.6 & -4.4 & 2.0 & 4.0 & 0 & 7.05 & 1.18 & 0.19 & 0.20 & 2.57 & 2.19 & 6.04 & 3 & 0 & 0 \\
\hline 824 & 8 & 6.5 & 85.8 & 0 & 10.8 & 6.0 & -1.6 & -1.0 & 3.0 & 5.3 & 0 & 6.94 & 1.62 & 0.29 & 0.27 & 2.68 & 2.73 & 5.82 & 5 & 0.10 & 0.0033 \\
\hline \multicolumn{22}{|c|}{ OPTICAL VIEW PORT TESTS } \\
\hline 834 & 9 & 6.5 & 85.7 & 0 & 13.6 & 7.3 & -0.1 & -0.1 & 3.5 & 5.9 & 0 & 6.94 & 1.80 & 0.51 & 0.39 & 2.75 & 3.02 & 5.72 & 9 & 0.14 & 0.018 \\
\hline 843 & N/A & 6.5 & 135.5 & 0 & 13.4 & 7.8 & -0.5 & -0.6 & 3.1 & 5.1 & 0 & 6.96 & 1.75 & 0.58 & 0.59 & 4.72 & 2.38 & 9.15 & 6 & 0 & 0 \\
\hline 849 & 11 & 6.5 & 135.4 & 0 & 13.1 & 9.0 & 3.6 & 3.4 & 7.2 & 8.6 & 0 & 6.97 & 4.19 & 0.96 & 1.40 & 5.43 & 4.73 & 9.24 & 15 & 0.18 & $\mathrm{~N} / \mathrm{A}$ \\
\hline 855 & N/A & 3.9 & 137.4 & 0 & 18.7 & 6.9 & -3.3 & -3.3 & 2.8 & 7.1 & 0 & 6.87 & 0.91 & 0.58 & 0.60 & 4.41 & 1.74 & 8.94 & 7 & 0 & 0 \\
\hline 861 & $\mathrm{~N} / \mathrm{A}$ & 4.0 & 133.8 & 0 & 17.4 & 7.6 & -1.4 & -1.4 & 5.5 & 9.1 & 0 & 7.05 & 1.90 & 0.64 & 0.68 & 4.32 & 2.59 & 9.10 & 7 & 0 & 0 \\
\hline 867 & 10 & 4.0 & 134.4 & 0 & 18.0 & 9.1 & 2.1 & 1.7 & 9.6 & 12.8 & 0 & 7.02 & 3.35 & 0.92 & 1.29 & 4.85 & 4.01 & 9.13 & 14 & 0.17 & 0.0069 \\
\hline
\end{tabular}




\section{Test Conditions - Wedge}

\begin{tabular}{|c|c|c|c|c|c|c|c|c|c|c|c|c|c|c|c|c|c|c|c|c|c|}
\hline 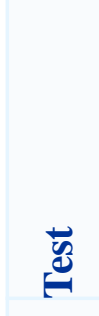 & 总 & 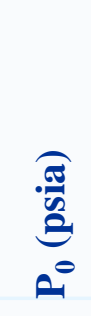 & 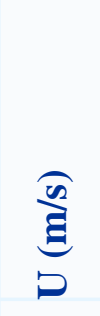 & 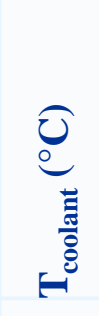 & $\begin{array}{c}0 \\
\underbrace{0}_{0} \\
\vdots \\
0 \\
0\end{array}$ & 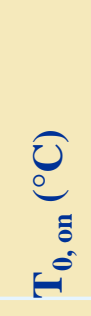 & 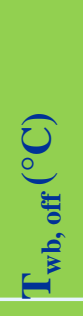 & $\underbrace{0}_{\substack{0 \\
0}}$ & $\underbrace{000}$ & 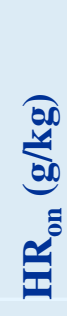 & 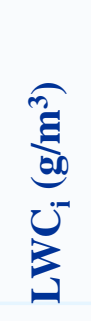 & 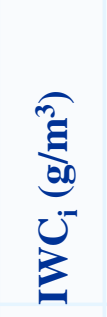 & 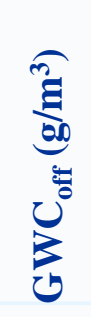 & 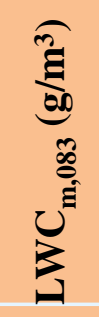 & 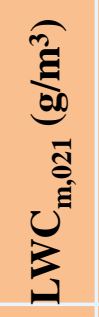 & 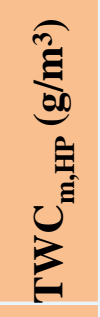 & 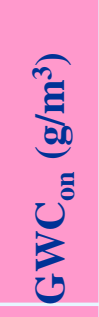 & 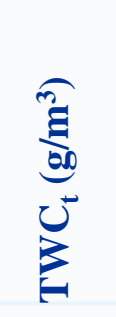 & $\begin{array}{l}\widehat{\widehat{\theta}} \\
\stackrel{a}{2}\end{array}$ & 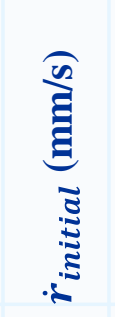 & 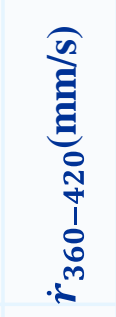 \\
\hline 883 & N/A & 6.5 & 87.1 & Adia & 19.9 & 10.6 & 3.5 & 2.5 & 4.2 & 6.9 & 0 & 6.84 & 2.14 & 0.88 & 0.80 & 3.18 & 3.42 & 5.56 & 16 & Trace & Trace \\
\hline 889 & N/A & 6.5 & 87.4 & Adia & 20.8 & 12.7 & 5.2 & 4.4 & 5.9 & 8.3 & 0 & 6.81 & 2.95 & 1.12 & 1.21 & 3.22 & 4.14 & 5.62 & 21 & 0 & 0 \\
\hline 901 & 15 & 6.5 & 86.9 & Adia & 20.5 & 8.3 & 3.5 & 2.5 & 4.0 & 7.9 & 0 & 16.68 & 2.04 & 1.85 & 2.15 & 5.71 & 3.87 & 14.84 & 13 & 0.123 & $\begin{array}{c}0.202 \\
*\end{array}$ \\
\hline 982 & 16 & 10.1 & 85.7 & -7 & 8.5 & 5.6 & 1.1 & 1.2 & 2.9 & 4.2 & 0 & 8.43 & 2.40 & 0.76 & 0.52 & 3.30 & 3.43 & 7.40 & 10 & 0.017 & 0.015 \\
\hline 996 & 17 & 10.0 & 83.9 & -5 & 8.2 & 4.3 & 3.2 & 2.1 & 4.9 & 5.6 & 0 & 8.61 & 3.88 & 1.28 & 1.03 & 3.62 & 4.32 & 8.17 & 16 & 0.048 & 0.021 \\
\hline 003 & 18 & 10.0 & 84.1 & -5 & 8.2 & 3.8 & 1.9 & 1.4 & 3.8 & 5.2 & 1.87 & 8.60 & 3.01 & 1.98 & 1.74 & 4.92 & 4.24 & 9.24 & 21 & 0.049 & 0.032 \\
\hline
\end{tabular}

